\title{
Empirical Modeling on Hot Air Drying of Fresh and Pre-treated Pineapples
}

\author{
Yardfon Tanongkankit ${ }^{1, a}$, Kanjana Narkprasom ${ }^{1}$, Nukrob Narkprasom ${ }^{1}$ \\ ${ }^{1}$ Maejo University in Chiang Mai, Thailand
}

\begin{abstract}
This research was aimed to study drying kinetics and determine empirical model of fresh pineapple and pre-treated pineapple with sucrose solution at different concentrations during drying. $3 \mathrm{~mm}$ thick samples were immersed into 30, 40 and 50 Brix of sucrose solution before hot air drying at temperatures of 60,70 and $80^{\circ} \mathrm{C}$. The empirical models to predict the drying kinetics were investigated. The results showed that the moisture content decreased when increasing the drying temperatures and times. Increase in sucrose concentration led to longer drying time. According to the statistical values of the highest coefficients $\left(R^{2}\right)$, the lowest least of chi-square $\left(\chi^{2}\right)$ and root mean square error (RMSE), Logarithmic model was the best models for describing the drying behavior of soaked samples into 30, 40 and 50 Brix of sucrose solution.
\end{abstract}

\section{Introduction}

Pineapple (Anana comosus) is a tropical fruit belonging to the Bromeliaceae family, and originated from South America [1]. This fruit has been reported to be low in calories and a source of nutritional components such as fiber, mineral and antioxidants [1-2]. Pineapple is commonly consumed in a form of fresh product. Nevertheless, it has a short storage life approximately 5 days at $4^{\circ} \mathrm{C}$ and 1 day at $15^{\circ} \mathrm{C}$ [3]. Therefore, to extend its shelf life pineapple is usually dried.

Drying is a process to remove moisture content in products to a level at which deterioration from chemical reaction and microorganism are minimized. However, this method involving in high temperatures and times can cause changes in final quality of products when compared to the fresh fruit [4]. To avoid this problem, pre-drying treatments such as addition of sugars are needed for fruit drying in order to the improvement in texture and the stability of the color pigment during storage [5].

Pre-treatment with sugar solution has been claimed to be very effective for producing high quality dried fruits and vegetable. Since sugar especially non-reducing sugar does not react with amino acids or proteins to cause Maillard reaction, browning and loss of nutrition in products can be eliminated [5]. Giovanna [6] reported that immersing pineapple in 50 Brix of sucrose solution for $30 \mathrm{~min}$ protected the color of pineapple during drying at 70,75 and $80^{\circ} \mathrm{C}$.

The objective of this study was to determine kinetic dryings of fresh pineapple and pre-treated pineapple with sucrose solution. Empirical modeling for describing drying behavior of pineapples was also investigated.

\section{Materials and Methods}

\footnotetext{
${ }^{\mathrm{a}}$ Corresponding author: yardfon@ hotmail.com
}

\subsection{Experimental material}

Fresh pineapple cv. Nanglae were obtained from local market in Chiang Mai. An initial moisture content of pineapple was $80 \pm 2 \%$ (d.b.), which was determined by drying the fresh pineapple in the electric thermal dryer at $105^{\circ} \mathrm{C}$ for $24 \mathrm{~h}$ until the weight was constant. Each run in the experiment was performed in triplicate.

\subsection{Sample preparation}

The fruits were hand peeled and sliced into a rectangular shape of $5 \times 10 \mathrm{~cm}$ and $5 \mathrm{~mm}$ thickness. A batch each of $250 \mathrm{~g}$ of slices were pre-treated accordingly with sucrose osmosis method by immersing fresh slices into sucrose solution (Food grade of $98 \%$ purity) with concentration of 30, 40 and 50 Brix for $30 \mathrm{~min}$. The samples were drained on a screen and reweighed.

\subsection{Drying experiment}

The prepared samples were dried in hot air dryer (Memmert, 500/108I, Germany). Approximately $250 \mathrm{~g}$ of the sample was placed as single layer on a perforated tray. The experiments were performed at temperature of 60,70 and $80{ }^{\circ} \mathrm{C}$. The samples were dried until a final moisture content of less than $18 \%$ (d.b.) was reached. During each experiment $3-5 \mathrm{~g}$ of the sample was taken out at various intervals to determine its moisture content. The moisture content of the sample was determined using a gravimetric method at $105^{\circ} \mathrm{C}[7]$.

\subsection{Mathematical modelling}


The moisture ratio (MR) of the pineapple was defined as follows [8]:

$$
M R=\frac{M_{t}-M_{e}}{M_{0}-M_{e}}
$$

Where $M_{\mathrm{t}}, M_{0}$ and $M_{\mathrm{e}}$ are the moisture content at any time of drying ( $\mathrm{kg}$ water $/ \mathrm{kg}$ dry mass), initial moisture content (kg water/ kg dry mass) and equilibrium moisture content, respectively. The experimental data at different thicknesses of paddy were fitted into 6 thin-layer drying models that commonly used in most food and biological materials (Table 1).

Table 1. Mathematical models applied the moisture ratio

\begin{tabular}{|l|l|c|}
\hline Model name & Model equation & Reference \\
\hline 1. Lewis & $M R=\exp (-k t)$ & {$[8]$} \\
\hline 2. Page & $M R=\exp \left(-k t^{n}\right)$ & {$[9]$} \\
\hline 3. Modified Page & $M R=\exp (-k t)^{n}$ & {$[9]$} \\
\hline 4.Wang and Singh & $M R=\operatorname{aexp}\left(-k t^{n}\right)+\mathrm{b} t$ & {$[10]$} \\
\hline $\begin{array}{l}\text { 5.Handerson and } \\
\text { Pabis }\end{array}$ & $M R=\mathrm{a} \cdot \exp (-k t)$ & {$[11]$} \\
\hline 6.Logarithmic & $M R=\operatorname{aexp}(-k t)+\mathrm{c}$ & {$[12]$} \\
\hline
\end{tabular}

\subsection{Correlation coefficients and error analyses}

The determination of coefficients $\left(R^{2}\right)$, reduced chisquare $\left(\chi^{2}\right)$ and root mean square error (RMSE) were used to evaluate the goodness fit. These parameters were calculated as follows:

$$
\begin{aligned}
& \chi^{2}=\frac{\sum_{i=1}^{N}\left(M R_{\text {exp }, \mathrm{i}}-M R_{\mathrm{pre}, \mathrm{i}}\right)^{2}}{N-z} \\
& R M S E=\sqrt{\left[\frac{1}{N} \sum_{i=1}^{N}\left(M R_{p r e, i}-M R_{\mathrm{exp}, j}\right)^{2}\right]}
\end{aligned}
$$

Where $N$ is the number of observations, $\mathrm{z}$ is the number of constants, $M R_{\exp }$ are the experimental and predicted moisture ratios, respectively.

\subsection{Statistical analysis}

The experiments were designed in complete random. The data were subjected to an analysis of variance (ANOVA) and are presented as mean values with standard deviations. Differences between mean values were established using Duncan's multiple range tests. Values were considered at a confidence level of $95 \%$. All statistical analyses were performed using SPSS ${ }$ software (version 17) (SPSS Inc., Chicago, IL). All experiments were performed in duplicate unless specified otherwise.

\section{Results and Discussion}

\subsection{Drying kinetics of pineapples}

Fig. 1 shows the drying curves of unpretreated and pretreated with different sucrose concentration samples. The results showed that the drying rates at higher temperatures were higher than those at lower temperatures. This is due to drying at higher temperature led to higher moisture diffusivity values and larger driving force for heat/mass transfer than at lower temperatures. The equilibrium moisture content (EMC) and time needed to dry a sample to the desired moisture content of less than $0.18 \mathrm{~g} / \mathrm{g}$ dry basis are listed in Table 2. EMC of the samples were in the range of 0.023-0.141 $\mathrm{g} / \mathrm{g}$ dry basis. It was also observed that higher concentration of sucrose resulted in longer drying time.
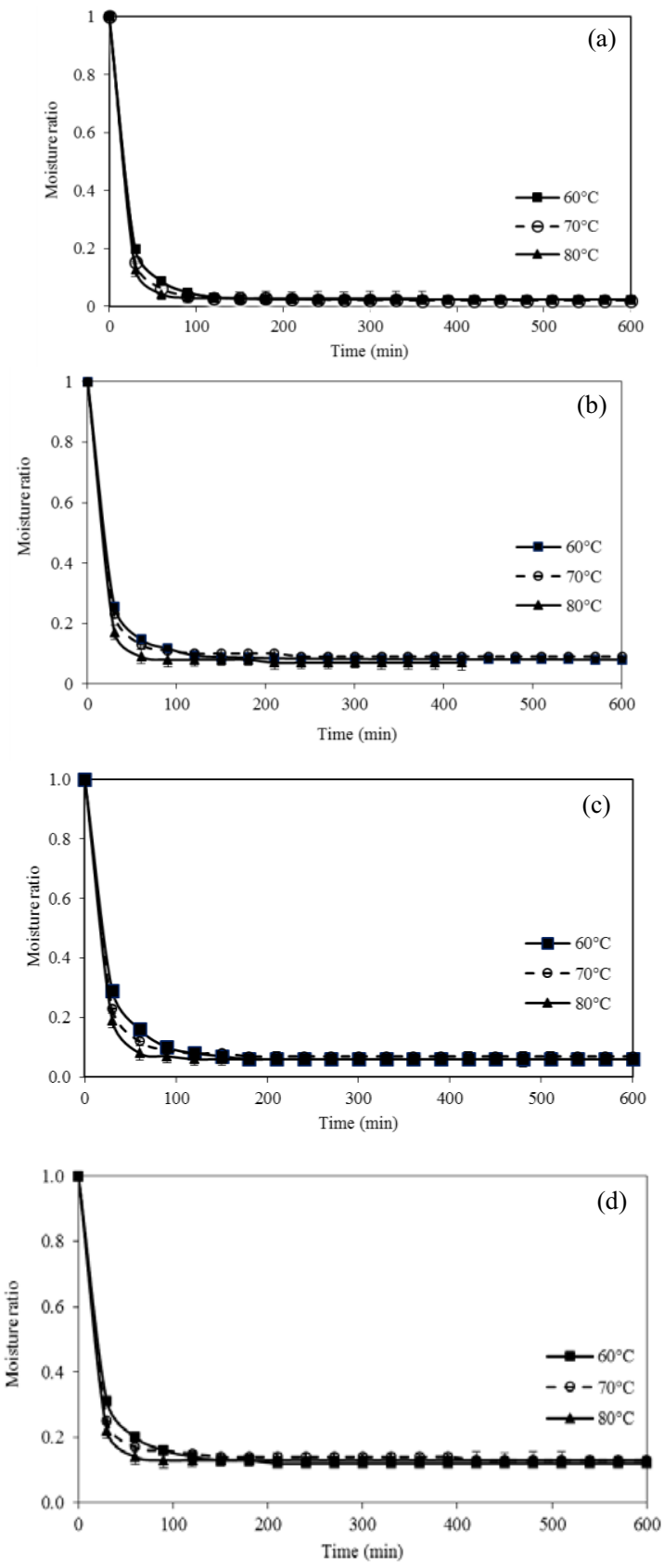

Figure 1. Drying curves of (a) unpretreated (b) pre-treated with 30 Brix of sucrose (c) pre-treated with 40 Brix of sucrose and (d) pre-treated with 50 Brix of sucrose samples during drying 
Table 2. Time to dry samples to the final moisture content of less than $18 \%$ dry basis.

\begin{tabular}{|c|c|c|c|}
\hline $\begin{array}{c}\text { Drying } \\
\text { temperature } \\
\left({ }^{\circ} \mathrm{C}\right)\end{array}$ & $\begin{array}{c}\text { Sucrose } \\
\text { concentration } \\
(\text { Brix })\end{array}$ & $\begin{array}{c}\text { Drying } \\
\text { time } \\
(\mathrm{min})\end{array}$ & $\begin{array}{c}\text { Equilibrium } \\
\text { moisture } \\
\text { content } \\
(\mathrm{g} / \mathrm{g} \mathrm{d.b} .)\end{array}$ \\
\hline \multirow{4}{*}{60} & 0 & 300 & 0.023 \\
\cline { 2 - 4 } & 30 & 720 & 0.080 \\
\cline { 2 - 4 } & 40 & 690 & 0.089 \\
\hline \multirow{4}{*}{70} & 50 & 900 & 0.075 \\
\cline { 2 - 4 } & 0 & 240 & 0.023 \\
\cline { 2 - 4 } & 30 & 540 & 0.093 \\
\hline \multirow{4}{*}{80} & 40 & 600 & 0.077 \\
\cline { 2 - 4 } & 50 & 810 & 0.122 \\
\cline { 2 - 4 } & 0 & 210 & 0.028 \\
\cline { 2 - 4 } & 30 & 300 & 0.073 \\
\hline
\end{tabular}

\subsection{Empirical modeling of drying curves}

Drying curves of the unpretreated and pre-treated pineapples under hot air at 60,70 and $80^{\circ} \mathrm{C}$ were fitted with six different moisture ratio models shown in Table 1. The statistical results of the different models, including the drying model coefficients and the comparison criteria used to evaluate goodness of fit, namely, $R^{2}, \chi^{2}$ and RSME, are summarized in Table 3 . The $R^{2}$ values for the models were in the rage of 0.7507-0.9997. $\chi^{2}$ values were varied between 0.0001 and 0.1081 and RMSE values between 0.122 and 0.981 . By comparing the criteria values among four drying models, it can be seen that Logarithmic equation was the best descriptive model for all treatments, since it exhibited the highest average value of $R^{2}$, the lowest average values of $\chi^{2}$ and RMSE. Thus, it was selected to represent the drying characteristics of pineapples.

Table 3. Statistical results obtained from different drying models.

\begin{tabular}{|c|c|c|c|c|c|c|}
\hline Model & $\begin{array}{c}\text { Sucrose } \\
\text { concentration (Brix) }\end{array}$ & $\begin{array}{c}\text { Temperature } \\
\left({ }^{\circ} \mathrm{C}\right) \\
\end{array}$ & Parameter & $R^{2}$ & $\chi^{2}$ & RMSE \\
\hline Newton & $\begin{array}{l}0 \\
30 \\
40 \\
50\end{array}$ & $\begin{array}{l}60 \\
70 \\
80 \\
60 \\
70 \\
80 \\
60 \\
70 \\
80 \\
60 \\
70 \\
80 \\
\end{array}$ & $\begin{aligned} \mathrm{k} & =0.0172 \\
\mathrm{k} & =0.0246 \\
\mathrm{k} & =0.0263 \\
\mathrm{k} & =0.0057 \\
\mathrm{k} & =0.0061 \\
\mathrm{k} & =0.0125 \\
\mathrm{k} & =0.0053 \\
\mathrm{k} & =0.0066 \\
\mathrm{k} & =0.0159 \\
\mathrm{k} & =0.0046 \\
\mathrm{k} & =0.0030 \\
\mathrm{k} & =0.0042\end{aligned}$ & $\begin{array}{l}0.9691 \\
0.9536 \\
0.9908 \\
0.7507 \\
0.8451 \\
0.8573 \\
0.7770 \\
0.8565 \\
0.8029 \\
0.8731 \\
0.8528 \\
0.9028 \\
\end{array}$ & $\begin{array}{l}0.0490 \\
0.0078 \\
0.0012 \\
0.0370 \\
0.0336 \\
0.0274 \\
0.0359 \\
0.0226 \\
0.0275 \\
0.0184 \\
0.0323 \\
0.0267 \\
\end{array}$ & $\begin{array}{l}0.0668 \\
0.0834 \\
0.0325 \\
0.1884 \\
0.1775 \\
0.1578 \\
0.1855 \\
0.1468 \\
0.1597 \\
0.1334 \\
0.1764 \\
0.1587 \\
\end{array}$ \\
\hline Page & $\begin{array}{l}0 \\
30 \\
40 \\
50\end{array}$ & $\begin{array}{l}60 \\
70 \\
80 \\
60 \\
70 \\
80 \\
60 \\
70 \\
80 \\
60 \\
70 \\
80 \\
\end{array}$ & $\begin{array}{l}\mathrm{k}=0.0527, \mathrm{n}=0.7182 \\
\mathrm{k}=0.1230, \mathrm{n}=0.5710 \\
\mathrm{k}=0.0649, \mathrm{n}=0.7554 \\
\mathrm{k}=0.3208, \mathrm{n}=0.2360 \\
\mathrm{k}=0.3170, \mathrm{n}=0.2366 \\
\mathrm{k}=0.3977, \mathrm{n}=0.2319 \\
\mathrm{k}=0.2779, \mathrm{n}=0.2578 \\
\mathrm{k}=0.0933, \mathrm{n}=0.4681 \\
\mathrm{k}=0.3741, \mathrm{n}=0.2589 \\
\mathrm{k}=0.0353, \mathrm{n}=0.6089 \\
\mathrm{k}=0.1257, \mathrm{n}=0.3572 \\
\mathrm{k}=0.1095, \mathrm{n}=0.3945\end{array}$ & $\begin{array}{l}0.9888 \\
0.9870 \\
0.9967 \\
0.9880 \\
0.9919 \\
0.9932 \\
0.9879 \\
0.9730 \\
0.9870 \\
0.8958 \\
0.9739 \\
0.9800 \\
\end{array}$ & $\begin{array}{l}0.0020 \\
0.0025 \\
0.0005 \\
0.0017 \\
0.0016 \\
0.0015 \\
0.0018 \\
0.0043 \\
0.0020 \\
0.0163 \\
0.0050 \\
0.0051 \\
\end{array}$ & $\begin{array}{l}0.0403 \\
0.0442 \\
0.0196 \\
0.0391 \\
0.0379 \\
0.0349 \\
0.0405 \\
0.0627 \\
0.0414 \\
0.1234 \\
0.0679 \\
0.0672 \\
\end{array}$ \\
\hline $\begin{array}{c}\text { Wang and } \\
\text { Singh }\end{array}$ & $\begin{array}{l}0 \\
30 \\
40 \\
50\end{array}$ & $\begin{array}{l}60 \\
70 \\
80 \\
60 \\
70 \\
80 \\
60 \\
70 \\
80 \\
60 \\
70 \\
80 \\
\end{array}$ & $\begin{aligned} \mathrm{k} & =0.0108, \mathrm{n}=2.84 \times 10^{-5} \\
\mathrm{k} & =0.0137, \mathrm{n}=4.49 \times 10^{-5} \\
\mathrm{k} & =0.0209, \mathrm{n}=1.18 \times 10^{-4} \\
\mathrm{k} & =0.0039 \mathrm{n}=4.53 \times 10^{-6} \\
\mathrm{k} & =0.0065, \mathrm{n}=1.15 \times 10^{-5} \\
\mathrm{k} & =0.0093 \mathrm{n}=2.47 \times 10^{-5} \\
\mathrm{k} & =0.0040, \mathrm{n}=4.82 \times 10^{-6} \\
\mathrm{k} & =0.0048, \mathrm{n}=6.45 \times 10^{-6} \\
\mathrm{k} & =0.0077, \mathrm{n}=1.60 \times 10^{-5} \\
\mathrm{k} & =0.0032, \mathrm{n}=2.94 \times 10^{-6} \\
\mathrm{k} & =0.0033, \mathrm{n}=2.95 \times 10^{-6} \\
\mathrm{k} & =0.0077, \mathrm{n}=1.60 \times 10^{-5}\end{aligned}$ & $\begin{array}{l}0.9328 \\
0.9058 \\
0.9925 \\
0.8688 \\
0.8618 \\
0.9132 \\
0.8816 \\
0.9195 \\
0.8611 \\
0.9185 \\
0.9230 \\
0.9072 \\
\end{array}$ & $\begin{array}{l}0.0188 \\
0.0316 \\
0.0014 \\
0.0331 \\
0.0318 \\
0.0317 \\
0.0306 \\
0.0170 \\
0.0184 \\
0.0170 \\
0.0213 \\
0.0334 \\
\end{array}$ & $\begin{array}{l}0.1093 \\
0.1325 \\
0.0298 \\
0.1667 \\
0.1558 \\
0.1420 \\
0.1597 \\
0.1273 \\
0.1663 \\
0.1216 \\
0.1351 \\
0.1598 \\
\end{array}$ \\
\hline Logarithmic & $\begin{array}{c}0 \\
30\end{array}$ & $\begin{array}{l}60 \\
70 \\
80 \\
60 \\
70 \\
80 \\
60 \\
70\end{array}$ & $\begin{aligned} & \mathrm{k}=0.9172, \mathrm{a}=121.16, \mathrm{c}=0.2513 \\
& \mathrm{k}=1.0168, \mathrm{a}=158.43, \mathrm{c}=0.1637 \\
& \mathrm{k}=1.0356, \mathrm{a}=443.81, \mathrm{c}=0.1621 \\
& \mathrm{k}=0.7307, \mathrm{a}=0.0268, \mathrm{c}=0.2677 \\
& \mathrm{k}=0.6968, \mathrm{a}=0.0328, \mathrm{c}=0.3032 \\
& \mathrm{k}=0.7395, \mathrm{a}=0.0438, \mathrm{c}=0.1621 \\
& \mathrm{k}=0.7228, \mathrm{a}=0.0249, \mathrm{c}=0.2750 \\
& \mathrm{k}=0.7806, \mathrm{a}=0.0164, \mathrm{c}=0.2326\end{aligned}$ & $\begin{array}{l}0.8735 \\
0.9249 \\
0.9271 \\
0.9998 \\
0.9997 \\
0.9991 \\
0.9998 \\
0.9981\end{array}$ & $\begin{array}{l}0.0320 \\
0.0207 \\
0.0146 \\
0.0001 \\
0.0001 \\
0.0003 \\
0.0000 \\
0.0003\end{array}$ & $\begin{array}{l}0.1527 \\
0.1175 \\
0.1032 \\
0.0053 \\
0.0106 \\
0.0151 \\
0.0053 \\
0.0167\end{array}$ \\
\hline
\end{tabular}




\begin{tabular}{|c|c|c|c|c|c|c|}
\hline & 50 & $\begin{array}{l}80 \\
60 \\
70 \\
80\end{array}$ & $\begin{array}{l}\mathrm{k}=0.7395, \mathrm{a}=0.0438, \mathrm{c}=0.1621 \\
\mathrm{k}=0.8355, \mathrm{a}=0.0092, \mathrm{c}=0.2015 \\
\mathrm{k}=0.7032, \mathrm{a}=0.0152, \mathrm{c}=0.3194 \\
\mathrm{k}=0.6720, \mathrm{a}=0.0190, \mathrm{c}=0.3479\end{array}$ & $\begin{array}{l}0.9991 \\
0.9922 \\
0.9968 \\
0.9962\end{array}$ & $\begin{array}{l}0.0003 \\
0.0012 \\
0.0006 \\
0.0010\end{array}$ & $\begin{array}{l}0.0151 \\
0.0330 \\
0.0237 \\
0.0291\end{array}$ \\
\hline $\begin{array}{l}\text { Henderson and } \\
\text { pabis }\end{array}$ & 30 & $\begin{array}{l}60 \\
70 \\
80 \\
60 \\
70 \\
80 \\
60 \\
70 \\
80 \\
60 \\
70 \\
80\end{array}$ & $\begin{array}{l}\mathrm{k}=0.9915, \mathrm{a}=0.0169 \\
\mathrm{k}=0.9950, \mathrm{a}=0.0244 \\
\mathrm{k}=0.9974, \mathrm{a}=0.0262 \\
\mathrm{k}=0.7369, \mathrm{a}=0.0029 \\
\mathrm{k}=0.8057, \mathrm{a}=0.0042 \\
\mathrm{k}=0.9115, \mathrm{a}=0.0101 \\
\mathrm{k}=0.7479, \mathrm{a}=0.0029 \\
\mathrm{k}=0.8793, \mathrm{a}=0.0050 \\
\mathrm{k}=0.9438, \mathrm{a}=0.0135 \\
\mathrm{k}=0.9248, \mathrm{a}=0.0039 \\
\mathrm{k}=0.7610, \mathrm{a}=0.0019 \\
\mathrm{k}=0.8322, \mathrm{a}=0.0030\end{array}$ & $\begin{array}{l}0.9696 \\
0.9538 \\
0.9027 \\
0.8584 \\
0.8854 \\
0.8742 \\
0.8707 \\
0.8870 \\
0.8149 \\
0.8875 \\
0.9082 \\
0.9298\end{array}$ & $\begin{array}{l}0.0054 \\
0.0089 \\
0.0231 \\
0.0208 \\
0.0231 \\
0.0260 \\
0.0203 \\
0.0183 \\
0.0278 \\
0.0167 \\
0.0182 \\
0.0185\end{array}$ & $\begin{array}{l}0.0662 \\
0.0832 \\
0.1373 \\
0.1385 \\
0.1423 \\
0.1459 \\
0.1363 \\
0.1288 \\
0.1543 \\
0.1250 \\
0.1301 \\
0.1277\end{array}$ \\
\hline Modified Page & 30 & $\begin{array}{l}60 \\
70 \\
80 \\
60 \\
70 \\
80 \\
60 \\
70 \\
80 \\
60 \\
70 \\
80\end{array}$ & $\begin{array}{l}\mathrm{k}=0.0166, \mathrm{n}=0.7182 \\
\mathrm{k}=0.0254, \mathrm{n}=0.5710 \\
\mathrm{k}=0.0267, \mathrm{n}=0.7554 \\
\mathrm{k}=0.0081, \mathrm{n}=0.2360 \\
\mathrm{k}=0.0077, \mathrm{n}=0.2366 \\
\mathrm{k}=0.0187, \mathrm{n}=0.2319 \\
\mathrm{k}=0.0069, \mathrm{n}=0.2578 \\
\mathrm{k}=0.0063, \mathrm{n}=0.4681 \\
\mathrm{k}=0.0224, \mathrm{n}=0.2589 \\
\mathrm{k}=0.0041, \mathrm{n}=0.6089 \\
\mathrm{k}=0.0030, \mathrm{n}=0.3572 \\
\mathrm{k}=0.0036, \mathrm{n}=0.3945\end{array}$ & $\begin{array}{l}0.9643 \\
0.9375 \\
0.9849 \\
0.8836 \\
0.8886 \\
0.8671 \\
0.8951 \\
0.9039 \\
0.8461 \\
0.9012 \\
0.9169 \\
0.9267 \\
\end{array}$ & $\begin{array}{l}0.0092 \\
0.0170 \\
0.0025 \\
0.0871 \\
0.1402 \\
0.0846 \\
0.0925 \\
0.0487 \\
0.0537 \\
0.0314 \\
0.1074 \\
0.1081 \\
\end{array}$ & $\begin{array}{l}0.0866 \\
0.1150 \\
0.0452 \\
0.2831 \\
0.3502 \\
0.2656 \\
0.2912 \\
0.2098 \\
0.0537 \\
0.1714 \\
0.3158 \\
0.3089 \\
\end{array}$ \\
\hline
\end{tabular}

\section{Conclusion}

The experiments were performed to determine drying characteristics of unpretreated pineapple and pre-treated pineapple with sucrose solution at 30,40 and 50 Brix. It was evidenced that higher drying temperature resulted in shorter drying time since moisture diffusivity values and larger driving force for heat and mass transfer were larger at higher drying temperatures Increase in sucrose concentration resulted in longer drying time. Different mathematical models were also determined with the drying behavior of pineapples. The results indicated that the Logarithmic model could present better predictions for the moisture transfer than others. Studies on physical and nutritional properties of the pineapple after pretreatment and drying are suggested as a future work.

\section{Acknowledgement}

The authors express their sincere appreciation to Upper Northen Research Administration network for supporting the study financially and Faculty of Engineering and Agro-Industry, Maejo University for laboratory equipment.

\section{References}

1. S. W. Wijeratnam, Ref. Modul. Food Sci. Ency. Food Health 380-384 (2015)

2. D. I. S. da Silva, G. D. R. Nogueira, A. G. Duzzioni, M. A. S. Barrozo, Indus. Crop. Product. 50 (2013)

3. S. Chen, B. Mulgrew, and P. M. Grant, IEEE Trans. on Neural Network. 4 (1993)

4. M. K. Krokida, V. T. Karathanos, Z. B. Maroulis, D. Marinos-Kouris, J. Food Eng. 59 (2002).

5. G. E. Lombard, J. C. Oliveira, P. Fito, A. Andre's, J Food Eng. 85 (2008)

6. G. Cortellino, P. Pani, D. Torreggiani, Procedia Food Sci., 1 (2011)

7. Association of Official Analytical Chemists (AOAC), Official methods of analysis, $15^{\text {th }}$ ed., The Association of Official Analytical Chemists: Washington D.C (1995).

8. I. Ceylan, M. Aktas, H. Dogan, Appl. Themal. Eng. 27 (2007)

9. M. Rasouli, S. Seiiedlou, H. R. Ghasemzadeh, H. Nalbandi,. Austra. J. Agri. Eng. 2 (2011)

10. C. Y. Wang, R. P. Singh. ASAE, (1978)

11. M. A. Basunia, T. Abe, J. Food Eng. 47 (2001)

12. Z. Wang, J. Sun, X. Liao, F. Chen, G. Zhao, Food Res. Inter. 40 (2007) 